\title{
Inducción de tolerancia en la hipersensibilidad a mesalazina (5-ASA)
}

\author{
L. VÁZQUEZ FUERTES, C. MARTÍNEZ-CÓCERA, T. ROBLEDO ECHARREN, \\ J. DOMÍNGUEZ ORTEGA, M. L. GONZÁLEZ GUTIÉRREZ
}

Servicio de Alergia. Hospital Clínico San Carlos. Madrid

INDUCTION OF TOLERANCE IN HYPERSENSITIVITY TO MESALAZINE (5-ASA)

\section{RESUMEN}

La mesalazina es un derivado del ácido 5-aminosalicílico (5-ASA, el cual es útil en el tratamiento de la enfermedad inflamatoria intestinal. La sulfasalazina está formada por dos partes, la sulfapiridina y el 5-ASA, siendo ésta última la parte activa de la molécula. Los nuevos preparados derivados del 5-ASA se desarrollaron tratando de evitar los efectos secundarios tradicionalmente asociados a la sulfapiridina, aunque se siguen observando y aparecen nuevos efectos.

Presentamos dos casos, el primero es un varón diagnosticado de enfermedad inflamatoria intestinal, con antecedentes de dos reacciones previas de urticaria y angioedema tras ácido acetilsalicílico, que presenta urticaria tras la toma de mesalazina. El segundo presenta urticaria generalizada tras tres meses de iniciar tratamiento con mesalazina. Dada la necesidad de tratamiento en ambos casos, se realiza el protocolo de desensibilización a mesalazina desarrollado en 17 días en nuestro servicio, con el que se llega a la tolerancia de dicho fármaco hasta dosis terapéuticas.

Ante pacientes con hipersensibilidad a distintos fármacos, que sean necesarios para el tratamiento de su patología, pueden realizarse pautas de "desensibilización", que aseguren una buena tolerancia.

PALABRAS CLAVE: Ácido 5-aminosalicílico (5-ASA). Desensibilización. Hipersensibilidad. Mesalazina.

\section{ABSTRACT}

Mesalazine is a derivative of 5-aminosalicylic acid (5-ASA), which is useful in the treatment of intestinal inflammatory disease. Sulfasalazine is formed by two parts, sulfapyridine and 5-ASA, the latter being the active part of the molecule. The new preparatives derived from 5-ASA were developed in an attempt to avoid the traditionally associated side effects to sulfapyridine, although they are still observed and new effects appear.

We present two cases. The first is a man diagnosed of inflammatory intestinal disease, with background of two previous reactions of urticaria and angioedema after acetyl salicylic acid, who presented urticaria after taking mesalazine. The second one had generalized urticaria after three months of initiating treatment with mesalazine. Given the need for treatment in both cases, a desensitization protocol to mesalazine was made. It was developed in 17 days in our service. Tolerance to that drug to therapeutic doses is reached.

When faced with patients with hypersensitivity to different drugs, that are necessary to treat their disease, "desensitization" regimes, that assure good tolerance, can be made.

KEY WORDS: 5-aminosalicylic acid (5-ASA). Desensitization. Hypersensitivity. Mesalazine.

Vázquez Fuertes L, Martínez-Cócera C, Robledo Echarren T, Domínguez Ortega J, González Gutiérrez ML. Inducción de tolerancia en la hipersensibilidad a mesalazina (5-ASA). An Med Interna (Madrid) 2005; 22: 586-588.

\section{INTRODUCCIÓN}

La sulfasalazina y los aminosalicilatos se usan en las formas leves-moderadas de los brotes de colitis ulcerosa $\mathrm{y}$ enfermedad de Crohn, y como tratamiento de mantenimiento para evitar recidivas. Los salicilatos son antiinflamatorios que inhiben LT B4, producen un bloqueo de la fagocitosis, disminuyen la IL-1, eliminan radicales libres de hidrógeno, inhiben al factor activador de plaquetas y la producción local de inmunoglobulinas, con disminución de la expresión de HLA en colon.
La sulfasalazina es el salicilato más antiguo y está constituido por dos moléculas: la sulfapiridina y el ácido 5-aminosalicílico (5-ASA o mesalazina o mesalamina).

A la sulfapiridina se le han atribuido la mayor parte de los efectos secundarios: reacciones de hipersensibilidad (5-50\%) (1), como urticaria, fiebre, artralgias, hepatitis, neutropenia, neumonitis, pancreatitis, hepatitis; reacciones dosis-dependiente, como naúseas, cefalea, y más raramente discrasias sanguíneas, anemia hemolítica, hepatitis, fenómeno de Raynaud, neurotoxicidad. También se ha descrito la oligospermia reversible tras retirada del tratamiento (2). 
Debido a ésto se han desarrollado nuevas moléculas que prescinden de ella, y que proceden del ácido 5-ASA. Se han visto, a pesar de esto, efectos secundarios de urticaria, angioedema, neumonitis, hepatitis o la aparición de otros en una mayor frecuencia (pancreatitis en la mesalazina con una frecuencia siete veces mayor que en la sulfasalazina) $(3,4)$.

Presentamos dos casos de "desensibilización" a la mesalazina en pacientes con urticaria en relación a su administración.

\section{CASOS APORTADOS}

Dos pacientes varones A y B, diagnosticados de enfermedad inflamatoria intestinal que presentaron urticaria generalizada tras varios meses de tratamiento con mesalazina. En el caso A, de 31 años, había presentado urticaria generalizada tras toma de mesalazina durante dos meses, así como reacciones previas de urticaria y angioedema tras toma de AAS (ácido acetilsalicílico). En el caso B, de 30 años, tras tres meses de tratamiento con mesalazina, presenta rash maculopapuloso generalizado, por lo que se retira. Seis meses más tarde se reinicia tratamiento con mesalazina, aunque había introducido tres meses antes budesonida vía oral y rectal. Tras otros tres meses de tratamiento con todo lo anterior y dos semanas después de la retirada de corticoides, presenta urticaria generalizada.

\section{Test cutáneos}

Se realizaron pruebas cutáneas según las recomendaciones de la Academia Europea de Alergología e Inmunología Clínica (EAACI) (5). Prick a $1 / 10$ y a $1 / 1$ con mesalazina $\left(10 \mathrm{mg} / \mathrm{ml}\right.$ de Claversal $\left.{ }^{\circledR}\right)$ y sulfasalazina $\left(10 \mathrm{mg} / \mathrm{ml}\right.$ de Salazopyrina $\left.{ }^{\circledR}\right)$. Se usó histamina a $1 / 10$ y $1 / 1$ como control positivo. El suero salino en prick se utilizó como control negativo. Los resultados se consideran negativos cuando la pápula obtenida es < o igual a $3 \mathrm{~mm}$ o menor que la histamina. Se realizaron prick en 10 pacientes sanos no expuestos a 5-ASA previamente.

\section{Provocación oral controlada con placebo a simple ciego}

Tras la toma de un placebo, se administran dosis crecientes del fármaco implicado en una reacción durante un tiempo determinado, a partir del cual se llega a la dosis deseada para el tratamiento, la cual se mantiene desde entonces.
Se deben descartar enfermedades o tratamientos que contraindiquen el uso de adrenalina, o enfermedades mal controladas (hipertensión arterial, diabetes).

Se deben suspender medicaciones que puedan interferir los resultados de las pruebas (corticoides, antihistamínicos, etc.).

$\mathrm{Si}$ aparecen reacciones durante la administración, como urticaria, angioedema, etc., se le administra el tratamiento preciso para revertirlas en cada caso y se suspende la provocación.

\section{"Desensibilización" a mesalazina}

Se pide consentimiento informado firmado por el paciente para la realización de pruebas cutáneas y de provocación oral.

Se llevó a cabo de forma ambulatoria el protocolo de desensibilización, elaborado en nuestro Servicio de Alergia como se puede observar en la Tabla I.

En el caso B, se suspendió la budesonida en enemas 7 días antes de iniciar la desensibilización .

Con este protocolo se administran en 17 días dosis (mg) crecientes de mesalazina, con varias dosis diarias, con un intervalo de tiempo que va aumentando cada día (45 minutos, 1 hora y 8 horas). Se administran dosis hasta alcanzar la tolerancia de la dosis terapéutica requerida por el paciente. Se señala en la tabla I la dosis diaria tolerada y la acumulada (la suma de los miligramos tolerados por el paciente hasta ese momento).

El día 17 se administran finalmente $1.000 \mathrm{mg} / 1.000 \mathrm{mg} / 1.000$ $\mathrm{mg}$ de mesalazina (3.000 $\mathrm{mg}$ al día).

Prick a $1 / 10$ y a $1 / 1$ con mesalazina y sulfasalazina fueron negativos en ambos pacientes y en los controles. Provocación oral en el paciente A con ácido acetilsalicílico hasta $500 \mathrm{mg}$, con urticaria localizada en cara. "Desensibilización" a mesalazina desarrollado en 17 días, con tolerancia de $1.000 \mathrm{mg}$ de mesalazina cada 8 horas (3.000 mg al día) en el paciente A y B. Desde entonces, y durante seis meses de seguimiento existe buena tolerancia a la mesalazina y mantienen el tratamiento con la misma dosis tolerada de forma continuada, para evitar la aparición de nuevas reacciones.

\section{DISCUSIÓN}

La frecuencia de reacciones adversas a los nuevos preparados derivados 5-ASA no ha disminuido, como se esperaba en un primer momento al atribuirse tradicionalmente la mayor parte de efectos secundarios a la sulfapiridina. Es más, con la mesalazina se ha observado un incremento de reacciones

TABLA I

PROTOCOLO DE DESENSIBILIZACIÓN A MESALAZINA

\begin{tabular}{lcccc}
\hline Días de administración & Dosis $(\mathrm{mg})$ & Intervalo entre dosis & $\begin{array}{c}\text { Dosis total diaria } \\
(\mathrm{mg})\end{array}$ & $\begin{array}{c}\text { Dosis acumulada } \\
(\mathrm{mg})\end{array}$ \\
\hline Día 1 & $0,5 / 1 / 2 / 4 / 8$ & 45 min & 15,5 & 15,5 \\
Día 2 & $15.5 / 30 / 60$ & 1 hora & 105,5 & 121 \\
Día 3 & $60 / 120 / 240$ & 1 hora & 420 & 541 \\
Día 4, 5, 6 y 7 & 500 & & 500 & 1.041 \\
Día 8 y 9 & $500 / 500$ & 8 horas & 1.000 & 2.041 \\
Día 10 y 11 & $500 / 500 / 500$ & 8 horas & 1.500 & 3.541 \\
Día 12,13 y 14 & $1.000 / 500 / 500$ & 8 horas & 2.000 & 5.541 \\
Día 15 y 16 & $1.000 / 1.000 / 500$ & 8 horas & 2.500 & 8.041 \\
\hline Día 17 & $1.000 / 1.000 / 1.000$ & 8 horas & 3.000 & 11.041 \\
\hline
\end{tabular}


adversas, tales como la pancreatitis, con respecto a la sulfasalazina, aunque los pacientes con hipersensibilidad previa a éste producto toleran 5-ASA hasta en un $80 \%$ (6).

Las reacciones de hipersensibilidad descritas con mesalazina son principalmente cutáneas (7-9) encontrándose en un rango similar que las reacciones cutáneas descritas por sulfasalazina (14\% en la mesalazina y $11 \%$ en la sulfasalazina); se han descrito también neumonitis $(10,11)$, hepatitis (12) y fiebre (13).

Estas reacciones de hipersensibilidad no son IgE dependientes como se deduce de la negatividad de las pruebas cutáneas, desconociéndose en la actualidad el mecanismo inmunológico que conduce a estas manifestaciones.

A pesar de las reacciones de hipersensibilidad, la mesalazina sigue siendo un tratamiento muy útil en la enfermedad inflamatoria intestinal. Por ello se han realizado otras "desensibilizaciones" con éxito, utilizando protocolos de distinta duración, desde 3 días, en un caso de anafilaxia por mesalazina, con toma conjunta con un AINE con ingreso hospitalario (14); hasta 32 días, en un caso de fiebre por mesalazina (13). Tambien se han llevado a cabo "desensibilizaciones" con otros compuestos 5-ASA (7) y diferentes vías de administración $(15,16)$, tanto en adultos como en niños y adolescentes (1).

Nuestra pauta se llevó a cabo en 17 días por vía oral, de forma ambulatoria con muy buenos resultados y comodidad para el paciente. Se destaca la importancia de no interrrumpir el tratamiento ya que podría necesitarse una nueva "desensibilización", con el consiguiente riesgo para el paciente.

Destacamos la eficacia y seguridad de la desensibilización a este grupo de fármacos en pacientes con reacciones de hipersensibilidad y que necesiten tratamiento por su patología. Esta eficacia y seguridad la proporcionan la evaluación, el control y el seguimiento por especialistas.

\section{Bibliografía}

1. Vasundhara T. Sulfasalazine desensitization in children and adolescents with chronic inflammatory bowel disease. AJG 1992; 87 (8): 10291032 .

2. Baker DE, Kane S. The Short- and Long-term safety of 5-aminosalicylate products in the treatment of ulcerative colitis. Rev Gastroenterol Disord 2004; 4:86-91.

3. Poldermans D, VanBlankenstein M. Pancreatitis induced by disodium azodisalicylate. Am J Gastroenterol 1988; 83: 578-580.

4. Ransford RAJ, Langmen MJS. Sulphasalazine and mesalazine: serious adverse reactions re-evaluated on the basis of suspected adverse reaction reports to the Committee on Safety of Medicines. Gut 2002; 51: 536-539.

5. Dreborg S, Frew A. Allergen standardization and skin tests. Position Paper EAACI subcommittee for allergen standardization and skin tests. Allergy 1993; 48 (Supl. 14): 49-82.

6. Turunen U, Elommaa I, Anttila VJ, Sëpäla K. Mesalazine tolerance in patients with inflammatory bowel disease and previous intolerance or alllergy to Sulphasalazine or Sulphonamides. Scand J Gastroenterol 1987; 22: 798-802.

7. Stelze RC, Squire EN. Oral desensitization to 5-aminosalicylic acid medications. Ann Allergy Asthma Innunol 1999; 83: 23-24.

8. Aparicio J, Carnicer F, Girona E. Cutaneous hypersensitivity reaction to

mesalazine. Am J Gastroenterol 1996; 91: 620-1.

9. Fardy JM, Lloyd DA, Reynolds RP. Adverse effects with oral 5-aminosalicyclic acid. J Clin Gastroenterol 1988; 10: 635-7.

10. Le Gros V, Saveuse H, Lesur G, Brion N. Luna and skin hypersensitivity to 5-aminosalicylic acid. BMJ 1991; 302: 970.

11. Sigal Sviri. Gafanovich, I, Mordechai R.Mesalamine-induced hypersensitivity pneumonitis. J. Clinical Gastroenterology 1997; 24: 3436.

12. Hautekeete M.L, Bourgeois N, Potvin P, et al. Hypersensitivity with hepatotoxicity to mesalazine after hypersensitivity to sulfasalazine. Gastroenterology 1992; 103: 1925-1927.

13. Gonzalo MA, Alcalde MM, García JM, Alvarado MI, Fernández L. Desensitization after fever induced by mesalazine. Allergy 1999; 54: 1224-1225.

14. Varela S, Díez MS, González C, González de la Cuesta C, Arenas L, Feijoo, Menéndez M. Oral desensitization to 5-ASA. Allergy 2002; 57: 371-372.

15. Smart BA, Adams CA, Lewis ST, Altman LC. Mesalamine desensitization for a patient with ulcerative colitis. Allergy Clin Immunol 1998; 101: S141.

16. Domínguez Ortega J, Martínez-Cócera C. RAM con salicilatos. A propósito de dos casos. Alergol Inmunol Clin 1998; 13 (Supl. 2): 198. 